\title{
Hypoglossia-hypodactyly syndrome with hydrocephalus: a clue to the aetiology?
}

\author{
Y Gillerot, L Van Maldergem, R Chef, L Koulischer
}

\begin{abstract}
A stillborn female with Hanhart's syndrome in association with hydrocephalus owing to stenosis of the aqueduct of Sylvius is presented. Neuropathological findings are suggestive of an acquired pathophysiological mechanism.
\end{abstract}

The association of terminal transverse defects and orofacial abnormalities has been subdivided by Temtamy and McKusick ${ }^{1}$ into several clinical entities. Hanhart's syndrome covers aglossia-adactyly, hypoglossia-hypodactyly, and peromelia with micrognathia. ${ }^{2}$ The aetiology of this rare syndrome remains unknown and environmental factors as well as genetic factors have been postulated. ${ }^{3}$ We report on a new case of this syndrome with central nervous system alterations suggestive of an acquired inflammatory origin.

\section{Case report}

This female infant was the third child of healthy, unrelated parents. The mother's second pregnancy ended at 30 weeks with the birth of a baby with left heart hypoplasia who survived a few days. The current pregnancy was uncomplicated until the birth at 36 weeks of a stillborn female baby with multiple malformations (fig 1). Birth weight was $2400 \mathrm{~g}$, length was $46 \mathrm{~cm}$, and head circumference $32 \mathrm{~cm}$. Clinical examination showed a proportionate large head with wide fontanelles. There were reduction anomalies involving both hands (severe hypoplasia of the second, third, and fourth rays owing to absence of the second and third phalanges) and feet (total absence of phalanges). Examination of the mouth showed

Institut de Morphologie Pathologique, Département: Clinical Genetics, Allée des Templiers 41, B-6280 Loverval, Belgium.

Y Gillerot, L Van Maldergem, L Koulischer

CHU André Vésale, Département: Maternity, Montignyle-Tilleul, Belgium.

R Chef

Correspondence to Dr Gillerot.

Received for publication 7 September 1990.

Revised version accepted for publication 30 November 1990 marked hypoplasia of the tongue (fig 1), which was closely adherent to the palate. A cleft palate was seen on dissection.

Macroscopic examination of the brain confirmed, after section, a slightly dilated ventricular system. Macroscopic examination showed an intense fibroblastic proliferation of meningeal tissue around the brainstem (fig 2a). Originating from this thickened meningeal envelope were small vessels penetrating deeply into the parenchyma inducing an astroglial reactional proliferation and ending in a necrotic calcified zone (fig $2 \mathrm{~b}$ ). The aqueduct of Sylvius was abnormal and showed a number of small channels with an intact ependymal lining. The cerebral hemispheres were thin and the cerebellum was normal.

Peripheral lymphocyte culture showed a normal 46, XX karyotype ( $R$ and $G$ banding).

\section{Discussion}

This stillborn female fulfils the criteria of aglossiaadactyly syndrome. ${ }^{1}$ Autosomal recessive inheritance has been postulated by Tuncbilek $e t a l^{4}$ in view of three reports of children born to consanguineous parents. Dellagrammaticas et $a l^{3}$ also suggested recessive inheritance, one of their two patients also being the product of a consanguineous marriage. Temtamy and McKusick ${ }^{1}$ suggested a dominant trait with variable expressivity since orofacial abnormalities, although less severe, were observed among the relatives of their reported cases. A dominant mutant gene was suggested by Nevin et al. ${ }^{5}$

However, Opitz, ${ }^{6}$ in a comment on the case of Dellagrammaticas $e t a l,{ }^{3}$ considered that there is, at present, no convincing evidence for considering this condition as a Mendelian trait and suggested a negligible recurrence risk should be given after the birth of a child with Hanhart's syndrome. Other reports implicate a secondary phenomenon for explaining this syndrome, such as environmental factors including drug intake during pregnancy, ${ }^{78}$ amniotic bands, and thromboembolic events. ${ }^{9}$ This last report was of three patients among whom were a discordant pair of monozygotic twins, an infant with an apple peel bowel, possibly secondary to an occlusion of the superior mesenteric artery, and a third child with a malformation complex also compatible with an intrauterine arterial occlusive event. 

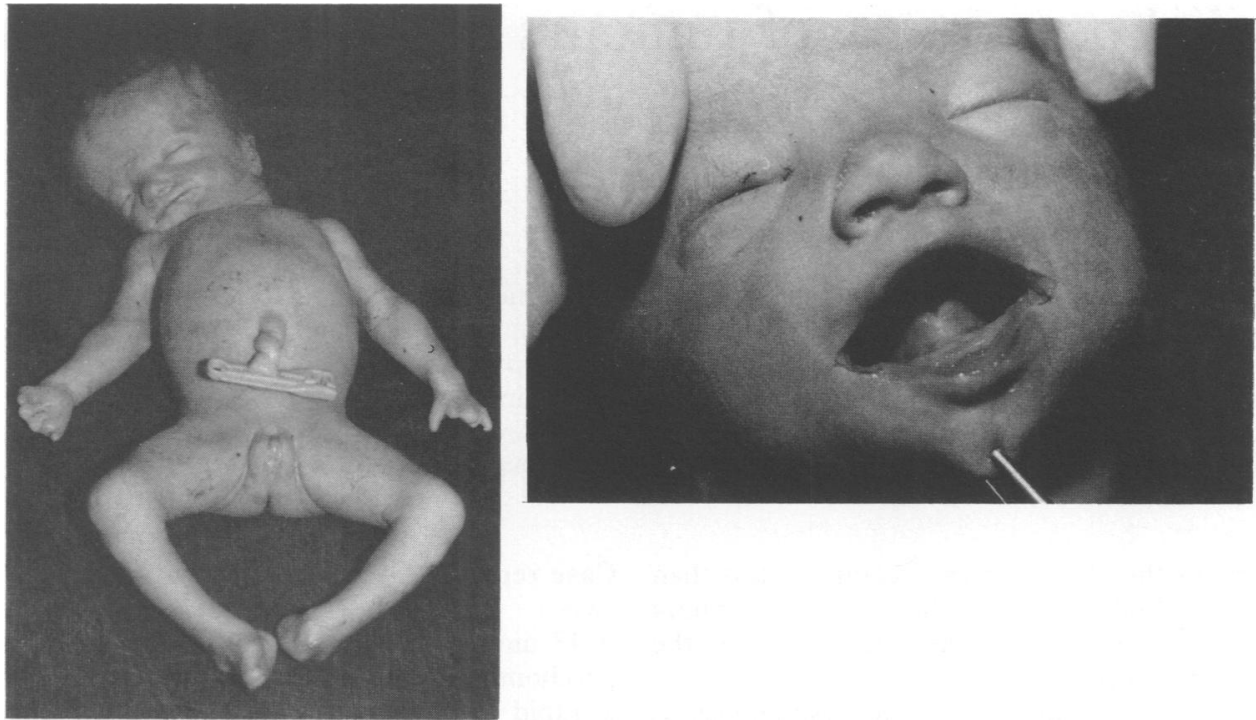

Figure 1 General appearance of the proband. Note hypoplastic tongue.

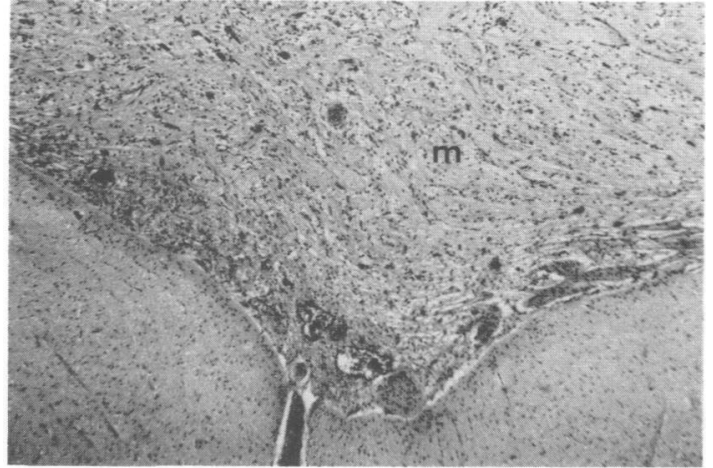

(a)

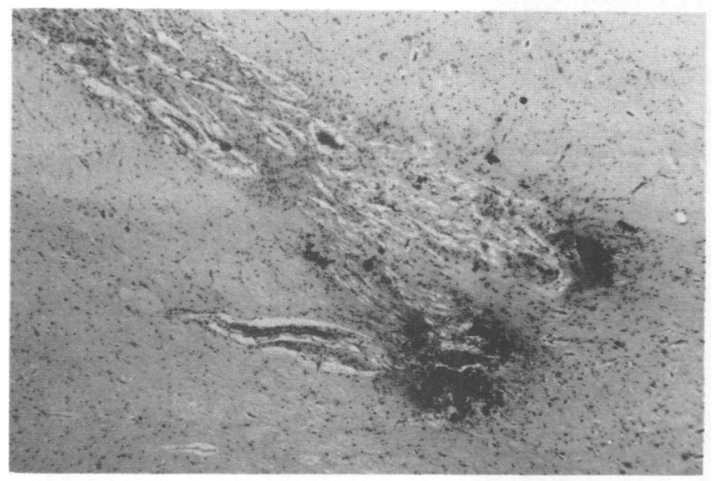

(b)

Figure 2 Microscopic examination of the brainstem, showing (a) important meningeal $(m)$ thickening and $(b)$ necrotic and calcified zones in the parenchyma $(H \mathcal{E} E)$.
Our case is, to the best of our knowledge, the first observation of Hanhart's syndrome associated with hydrocephalus and neuropathological changes. One could hypothesise the coincidental occurrence of Hanhart's syndrome and acquired hydrocephalus or, conversely, the association of these two conditions as a consequence of a similar causative agent is another possibility. The reported cases with vascular involvement mentioned above support the last hypothesis in which limb buds, tongue, and brain would be subject to the same vascular changes, thus leading to the syndrome described here.

The authors would like to thank Professor G Lyon (UCL, Brussels) for neuropathological data on this case.

1 Temtamy S, McKusick VA. The genetics of hand malformations. Birth Defects 1978;XIV(3):73-81.

2 Hermann J, Pallister RD, Gilbert EF, et al. Studies of malformation syndromes of man. XXXXI-B. Nosologic studies in the Hanhart and the Möbius syndrome. Eur $\mathcal{F}$ Pediatr 1976;122: $19-55$.

3 Dellagrammaticas H, Tzaki M, Kapiki A, et al. Hanhart syndrome: possibility of autosomal recessive inheritance. In: Skeletal dysplasias. New York: Alan R Liss, 1982:299-304.

4 Tuncbilek E, Yallin C, Atasu M. Aglossia-adactylia syndrome (special emphasis on the inheritance pattern). Clin Genet 1977;11:421-3.

5 Nevin NC, Burrows D, Allen G, Kernohan DC. Aglossiaadactylia syndrome. $\mathcal{F}$ Med Genet 1975;12:89-93.

6 Opitz JM. Comment. In: Skeletal dysplasias. New York: Alan R Liss, 1982:305.

7 Hall BD. Aglossia-adactylia. Birth Defects 1971;VII(7):233-6.

8 Bökesoy I, Akzüyek G, Deniz E. Oromandibular limb hypogenesis/Hanhart's syndrome. Clin Genet 1983;9:24-47.

9 Robinow M, Marsh JL, Edgerton MT, Sabio H, Johnson GF. Discordance in monozygotic twins for aglossia-adactylia, and possible clues to the pathogenesis of the syndrome. Birth Defects
$1978 ; X I V(6 a): 223-30$. 